\title{
Desafios de Pesquisa em Arquiteturas para IoT social
}

\author{
Leandro Camargo ${ }^{1}$, Ana Marilza Pernas ${ }^{1}$, Adenauer Yamin ${ }^{12}$, Felipe Haertel ${ }^{2}$ \\ ${ }^{1}$ Universidade Federal de Pelotas - UFPEL, Pelotas, Brasil \\ ${ }^{2}$ Universidade Católica de Pelotas - UCPEL, Pelotas, Brasil \\ \{leandro.camargo, marilza, adenauer\}@inf.ufpel.edu.br, \\ felipe@haertel.com.br
}

\begin{abstract}
Social IoT applications, with their different natures, present several conception challenges, architecture definition, implementation, and relationship management. This article conducts a Systematic Literature Review - RSL, where it selects recent research in this area, our aim is to architectural features investigate and existing research gaps. As the main contribution of this study, we would highlight the architectural aspects for a Social IoT solution, we also group studies with an emphasis on a particular theme, guiding future area research and favoring new solutions development. Finally, a contributions summary of works selected is described.
\end{abstract}

Resumo. As aplicações em IoT Social, com suas diferentes naturezas, apresentam vários desafios na concepção, definição da arquitetura, implementação $e$ gerenciamento das relações. Este artigo realiza uma Revisão Sistemática de Literatura - RSL, onde seleciona as pesquisas recentes nesta área, com intuito de investigar as características arquiteturais e as lacunas de pesquisa ainda existentes. Como principal contribuição deste estudo, destacaríamos os aspectos arquiteturais mais importantes para uma solução Social IoT, bem como o agrupamento dos estudos que dão ênfase a determinada temática, isso pode guiar futuras pesquisas na área e favorecer o desenvolvimento de novas soluções. Por fim, uma síntese das contribuições dos trabalhos selecionados está descrita.

\section{Introdução}

A Internet das Coisas - Internet of Things, IoT - é um arranjo de objetos interconectados, dispositivos eletromecânicos que possuem a capacidade de processamento e transmissão autônoma dos dados, com o uso de tecnologias de comunicação sem a necessidade do envolvimento humano. A IoT é constituída por sensores, máquinas, pessoas e coisas, visando atingir a conexão entre: pessoas; coisas; pessoas e coisas, oferecendo alguma funcionalidade [Bai et al. 2020], estando estruturada para a oferta de informação direcionada, o controle remoto e o gerenciamento inteligente, para tal, sua funcionalidade mais básica é a conectividade onipresente.

No contexto da Internet das Coisas Social (SIoT), que constitui uma frente de estudo da IoT, os dispositivos heterogêneos não apenas se conectam e interagem, mas também socializam e colaboram entre si para realizar tarefas específicas [Afzal et al. 2019]. Contudo, essa troca de informações entre os objetos traz 
muitas implicações, as quais se desdobram em diferentes desafios de pesquisa, pois se trata de um ecossistema digital altamente interconectado que deve prover suporte a coleta de dados e a troca de comandos de atuação em uma perspectiva de elevada escalabilidade.

Devido à natureza all-in-one - tudo em um - da SIoT, o seu design arquitetônico, implementação, gerenciamento operacional e manutenção estão levantando preocupações, dentre as quais muitas são desafiadoras aos pesquisadores, acadêmicos, engenheiros, organismos de padronização e outros participantes do mercado [Rho and Chen 2019]. Logo, o futuro da IoT não apenas considera as capacidades de cognição humana evoluindo para um ecossistema sábio e com alma, mas também aprende muito com os princípios da sociedade [Ning et al. 2016].

Assim, o presente artigo tem como objetivo central sistematizar os desafios de pesquisa presentes quando da concepção de arquiteturas para provimento de uma SIoT. Nesse contexto, foi realizada uma Revisão Sistemática de Literatura - RSL - dos trabalhos publicados em periódicos indexados que destaquem a implementação de características arquiteturais oportunas para o escopo da SIoT.

A contribuição central do trabalho, deste modo, está na identificação das características arquiteturais que podem suportar os processos fundamentais da IoT - percepção abrangente, transmissão confiável, processamento inteligente. Como principal resultado é apresentado um resumo sistematizado do estado-da-arte e dos desafios de pesquisa ainda presentes na concepção de arquiteturas voltadas à Social IoT.

O restante do artigo está estruturado da seguinte maneira, a Seção 2 aborda os fundamentos básicos voltados à temática desta pesquisa. A Seção 3 destaca a metodologia utilizada na Revisão Sistemática de Literatura. Já a Seção 4 expõe alguns resultados em alinhamento às questões de pesquisa, facilitando o entendimento dos desafios arquiteturais em SIoT. Por fim, a Seção 5 destaca os obstáculos e aspectos que precisam ser tratados pelas futuras arquiteturas.

\section{Fundamentos básicos em SIoT}

Com intuito de fornecer os fundamentos acerca da SIoT e os outros conceitos gerais abordados neste estudo, principalmente os relacionados com as questões estruturais e das funcionalidades presentes nas arquiteturas desse domínio, esta seção destaca algumas publicações científicas que são referência na área, bem como os conceitos que auxiliam na compreensão dos objetivos deste trabalho.

\subsection{Relacionamentos em um ambiente inteligente}

$\mathrm{Na}$ infraestrutura provida pela IoT os objetos têm a capacidade de observar e ouvir, uns aos outros. Já no ambiente de SIoT, além disso, eles também socializam informações com os componentes da rede, visando obter melhores resultados em problemas complexos por meio da colaboração e cooperação mútua [Lippi et al. 2018].

Em um ambiente de SIoT, a rede social pode ser estabelecida em diferentes níveis, considerando os relacionamentos construídos pelos componentes envolvidos [Roopa et al. 2019]. Exemplificando, os seres humanos constituem relações sociais com base em vários fatores, tais como objetivos em comum, interesse, amizade, enquanto também estão ligados ao objeto físico por meio de relacionamentos, estabelecidos em 
função da necessidade de uso ou da posse. No contexto dos objetos físicos, eles estabelecem relacionamentos entre si com base em características como o nível de interação, as atividades chave e a localização no ambiente inteligente.

\subsection{Arquitetura básica orientada à Internet das Coisas}

Os modelos arquiteturais na IoT usualmente seguem como base um esquema de três camadas: (i) percepção, que é dedicada a aquisição de dados e colaboração entre nodos conectados; (ii) rede, a qual possibilita a transferência de dados através de diferentes tipos de conexão, muitas vezes em regime de mobilidade; (iii) aplicação, onde os sistemas de IoT são implantados juntamente com as funcionalidades de um servidor em nuvem ou middleware [Saleem et al. 2016]. Contudo, esse modelo deixa de atender um componente fundamental da SIoT que trata da gerência dos relacionamentos, tal função permite o envolvimento do objeto em atividades de socialização.

Desta forma, um modelo arquitetural para SIoT precisa incluir funcionalidades como a descoberta de serviços e o gerenciamento das relações impondo assim, novos desafios estruturais. Nesse sentido, foi proposto por [Cirani et al. 2014] uma arquitetura escalável e auto-configurável, de ponto a ponto para redes IoT de larga escala. Como principal contribuição desta arquitetura, está a oferta de serviços automatizados e mecanismos de descoberta de recursos, sem a necessidade de intervenção humana na configuração.

\subsection{Internet das Coisas Social (SIoT)}

A SIoT difere das redes sociais e da IoT em três aspectos principais: (i) a SIoT estabelece e explora relações sociais entre coisas, independente da posse ou interferência de pessoas, as quais podem estar envolvidas na mediação, mas as relações são estabelecidas pelas coisas; (ii) o objeto pode descobrir recursos e serviços por si, através das relações sociais firmadas no ambiente IoT, reduzindo assim, a carga cognitiva; (iii) a SIoT não é pautada em interfaces que dependam da manipulação humana, em vez disso, é uma plataforma para serviços de rede social, lidando com objetos que interoperam autonomamente [Saleem et al. 2016].

A convergência da computação e da comunicação centradas no social, permitirá que os dispositivos IoT aproveitem o contexto para a otimização dos serviços oferecidos e/ou personalização das entregas [Dhelim et al. 2021]. Um fator motivacional na consolidação da SIoT é a crescente presença de dispositivos inteligentes transportados junto aos usuários de ambientes hiper-conectados. Também discute a relação entre o contexto desse usuário que circula no ambiente e o compartilhamento de informações [Al-Turjman 2019].

Com base nas questões apresentadas, os desafios de socialização na Internet das Coisas vão desde a descoberta e composição de serviços, navegabilidade no ambiente, gestão do relacionamento e confiança, as quais ainda são lacunas de pesquisa. Por este motivo, o presente estudo realiza uma análise das características estruturais das arquiteturas, com foco central na gestão dos relacionamentos estabelecidos entre os componentes de ambientes inteligentes, detalhadas nas próximas seções.

\section{Revisão Sistemática de Literatura}

O método selecionado para a organização e estruturação da Revisão Sistemática de Literatura - RSL, está orientado pelos procedimentos definidos por Petersen 
[Petersen et al. 2008]. O objetivo da RSL é de agregação das informações relevantes encontradas em relação ao objeto de pesquisa, apresentando como resultado uma síntese da área, a qual é oriunda de diferentes fontes tendo como base a análise de estudos anteriores.

Para contemplar o propósito central deste artigo na oferta de uma visão geral da literatura que aponte as características arquiteturais das soluções IoT no domínio social, conforme exposto na Figura 1 a RSL está distribuída em três etapas: (i) preparação inicial da pesquisa com a composição de um arcabouço teórico identificando conceitos importantes e taxonomias do domínio; (ii) definição das questões de pesquisa, montagem e validação da expressão de busca e triagem dos artigos selecionados; (iii) análise qualitativa dos dados, traçando relações entre funcionalidades e características arquiteturais.

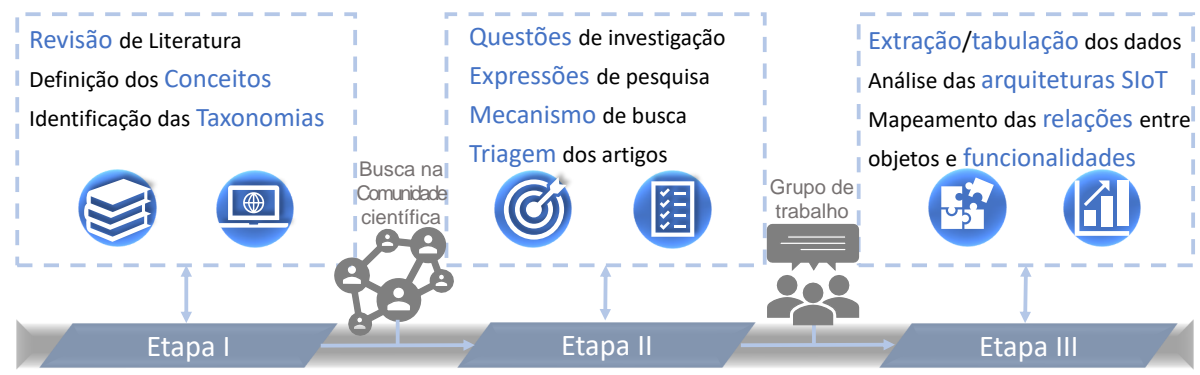

Figura 1. Esquema consolidado da metodologia adotada na RSL

Entre as etapas aplicadas na RSL foram realizadas reuniões do grupo para apresentação dos resultados parciais e planejamento da etapa seguinte. O resultado da triagem dos artigos passou pela análise e validação de um segundo membro da equipe.

\subsection{Estratégias de busca}

Na tentativa de validação do termo principal destinado à seleção dos artigos, bem como a delimitação temporal da busca foi utilizada a ferramenta do Google Trends ${ }^{1}$ que permite medir a popularidade dos assuntos pesquisados mundialmente nos buscadores da WEB. A Figura 2 apresenta o gráfico, no qual o eixo horizontal corresponde ao período temporal, já o vertical aponta para o volume de buscas.

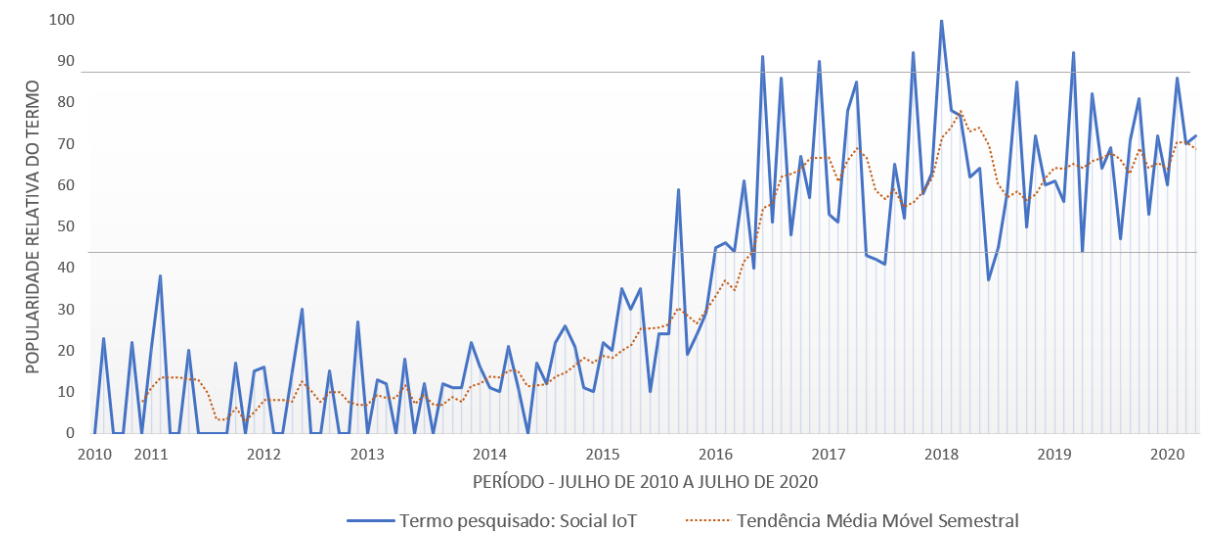

Figura 2. Interesse de consulta mundial na WEB para o termo Social loT

\footnotetext{
${ }^{1}$ https://trends.google.com/trends
} 
Com o suporte da Figura 2 é percebido um interesse mais acentuado pelo tema a partir do ano de 2017. Logo, este indicador serviu como critério de inclusão dos artigos, limitando a obtenção de obras publicadas nos anos de 2017 até 2020, além da restrição quanto ao idioma de publicação, apenas obras escritas em Inglês.

Já quanto aos critérios de exclusão foram definidos os seguintes: (i) livros, devido à dificuldade de acesso; (ii) s ites, pois a prioridade das fontes são jornais, revistas, anais ou proceedings de eventos científicos da área; (iii) baixa citação, foram selecionados apenas artigos referenciados por mais de um estudo, demonstrando alguma relevância e; (iv) o termo trust, pelo fato de apresentar um foco aprofundado em questões de segurança e, conforme observado em leituras prévias do assunto, normalmente, as pesquisas abordam funcionalidades específicas da área sem alteração da arquitetura existente.

Baseado nos primeiros resultados houve a necessidade de inclusão dos termos taxonomy, features e architecture, na composição da expressão de busca. Assim, foi observada a seleção de artigos mais assertivos com maior potencial para contribuir na presente pesquisa.

\subsection{Mecanismo de pesquisa}

Esta etapa compreendeu a escolha do mecanismo de busca acadêmica e os bancos de dados bibliográficos destinados à pesquisa. A escolha do repositório pode moldar diferencialmente o resultado das revisões sistemáticas [Cumpston et al. 2019]. Isso ocorre porque existem certos bancos de dados, como o Google Scholar ${ }^{2}$, que são mais amplos e abrangentes que outros, como o SciELO ${ }^{3}$ - Scientific Electronic Library Online.

Em pesquisa, 12 mecanismos de busca amplamente utilizados foram comparados, apontando que o Google Scholar oferece o maior volume de informações acadêmicas, ao manter cerca de 389 milhões de registros [Gusenbauer 2019]. Isso o torna um mecanismo de pesquisa acadêmico bastante abrangente e, por esse motivo, se optou pelo emprego desse indexador para o rastreio e seleção dos trabalhos correlatos.

\subsection{Triagem dos artigos}

Cumprida as etapas de definição das regras e filtros adequados à pesquisa foi construída a seguinte expressão de busca: \{ "taxonomy" AND "features" AND "architecture" AND "social internet of things" -book -trust-site \}. A seleção dos artigos ocorreu no mês de julho de 2020, através do indexador Google Scholar, que gerou um recorde de trinta e quatro (34) documentos, destes, dezesseis (16) não apresentavam citações e, por esse motivo, eles foram posteriormente descartados. Já os demais dezoito (18) artigos, ocorreram a partir dos repositórios da Elsevier, IEEE, IET, MDPI, PKP e Springer.

Fica evidente na Tabela 1 a variedade de bibliotecas digitais acessadas para a obtenção dos artigos. De certa forma, isso promove um melhor entendimento da produção gerada por diferentes grupos de pesquisa com publicações em diversos eventos e com ampla cobertura geográfica.

\footnotetext{
${ }^{2}$ https://scholar.google.com/

${ }^{3}$ https://scielo.org/en
} 
Tabela 1. Distribuição de artigos por veículo e volume de citações

\begin{tabular}{c|c|c}
\hline Veículo & $\mathrm{N}^{\mathbf{o}}$ Artigos & $\mathrm{N}^{\circ}$ Citações \\
\hline Elsevier & 3 & 017 \\
IEEE & 7 & 204 \\
IET & 1 & 004 \\
MDPI & 2 & 016 \\
PKP & 1 & 002 \\
Springer & 4 & 104 \\
\hline
\end{tabular}

\subsection{Questões de pesquisa}

A questão de pesquisa principal abordada na investigação foi deliberada como: "Quais são as características arquiteturais presentes em soluções SIoT?”. Para responder a esta indagação generalista, houve a derivação em outras três questões específicas $(\mathrm{QE})$, conforme segue:

- QE1 - A pesquisa apresenta uma arquitetura?

- Permite a identificação do foco aplicado ao estudo.

- QE2 - Quais funcionalidades-chave foram atendidas?

- Demonstra a complexidade do projeto.

- QE3 - Como ocorre o relacionamento entre os objetos?

- Cobre a forma como são tratadas as relações no ambiente.

A próxima seção conduz um apanhado dos trabalhos selecionados, onde são destacadas as funcionalidades-chave implementadas pela solução, o nível de relação estabelecido entre os objetos no ambiente inteligente, bem como, a abordagem arquitetural presente nos estudos que foram elencados conforme os parâmetros estabelecidos na revisão sistemática de literatura.

\section{Oportunidades de pesquisa}

Esta seção analisa os recentes esforços de pesquisa direcionados à arquitetura de uma SIoT no cenário computacional oferecido pela Internet das Coisas. Nesse sentido, a Figura 3 sintetiza os aspectos destacados pelos artigos selecionados na RSL realizada neste estudo. Tais aspectos são desdobramentos de pesquisa que integram uma estrutura arquitetônica ideal voltada à Social IoT.

\begin{tabular}{|c|c|c|c|c|c|}
\hline Interoperabilidade & Segurança & $\begin{array}{l}\text { Gestāo de } \\
\text { Interferências }\end{array}$ & $\begin{array}{l}\text { Controle de } \\
\text { Recursos }\end{array}$ & $\begin{array}{c}\text { Consciència } \\
\text { Energética }\end{array}$ & $\begin{array}{l}\text { Qualidade do } \\
\text { Serviço (QoS) }\end{array}$ \\
\hline $\begin{array}{l}\text { Maghawry; Ghoniemy } 2019 \\
\text { Seal; Mukherjee } 2018\end{array}$ & $\begin{array}{l}\text { Tahsien et al. } 2020 \\
\text { Wu et al. } 2017\end{array}$ & $\begin{array}{l}\text { Seal; Mukherjee } 2018 \\
\text { Kolozali et al. } 2018\end{array}$ & $\begin{array}{l}\text { lqbal et al. } 2018 \\
\text { Loscri et al. } 2018\end{array}$ & $\begin{array}{l}\text { Tahsien et al. } 2020 \\
\text { Anke } 2019 \\
\text { Anjomshoa et al. } 2017\end{array}$ & $\begin{array}{l}\text { Cicirelli et al. } 2019 \\
\text { Armando et al. } 2018 \\
\text { lqbal et al.2018 }\end{array}$ \\
\hline
\end{tabular}

Figura 3. Aspectos a considerar no projeto de arquiteturas Social loT

As pesquisas de [Anjomshoa et al. 2017], [Anke 2019], [Seal and Mukherjee 2018], [Maghawry and Ghoniemy 2019], [Loscri et al. 2018], [Iqbal et al. 2018] e [Wu et al. 2017] abordam arquiteturas com presença do middleware - elemento intermediário - que fornece funcionalidades e recursos comuns, voltados à composição de serviços em ambientes inteligentes. 
Os trabalhos de [Cicirelli et al. 2019], [Kolozali et al. 2018] e [Armando et al. 2018] propõem uma arquitetura focada na gestão dos relacionamentos estabelecidos entre os objetos. Embora o artigo de [Tahsien et al. 2020], não apresente de forma explícita uma arquitetura, ele propõe uma integração de Inteligência Artificial - IA, orientada à detecção de comportamentos anormais, originários de algum objeto malicioso, a qual pode complementar as arquiteturas existentes.

Assim, as funcionalidades-chave incorporadas às arquiteturas do paradigma do SIoT requerem atenção da comunidade industrial e acadêmica, para o enfrentamento dos obstáculos e, ainda, para impulsionar o desenvolvimento de aplicativos atraentes e amplamente aceitos. Tais funcionalidades podem ser classificadas em diferentes frentes de pesquisa, como: gestão de relacionamentos (GR), descoberta de serviços (DS), composição de serviços (CS) e gerenciamento de confiança (GC) [Afzal et al. 2019].

Outro desafio fica a cargo da gestão adequada das relações estabelecidas entre os objetos que integram os ambientes inteligentes. Para [Roopa et al. 2019] os relacionamentos em um sistema SIoT podem ser classificados em dois tipos: relacionamento usuário-objeto (UO), onde existe alguma forma de associação entre o usuário e o objeto físico. Já no relacionamento objeto-objeto (OO), os objetos físicos estão ligados entre si por meio de alguma relação de conveniência, similaridade ou composição de novos serviços.

A Tabela 2 resume os artigos analisados, em respostas às três questões específicas dessa pesquisa, sob o ponto de vista arquitetônico - QE1, bem como, das funcionalidades presentes - QE2, além disso, quanto ao tipo de relacionamento estabelecido entre os objetos - QE3.

Tabela 2. Funcionalidades arquiteturais destacadas nos artigos

\begin{tabular}{l|l|l|l|l}
\hline Autor & QE1 & QE2 & QE3 & Foco \\
\hline [Anjomshoa et al. 2017] & Sim & CS & UO & Serviços baseado em IA \\
[Anke 2019] & Sim & CS & OO & Serviços baseado em IA \\
[Seal and Mukherjee 2018] & Sim & CS & OO & Arquitetura Fog \\
[Maghawry and Ghoniemy 2019] & Sim & CS/DS & UO & Sensores integrados SN \\
[Loscri et al. 2018] & Sim & CS/GR & OO & Análise motriz social \\
[Iqbal et al. 2018] & Sim & CS/GR & OO & Serviços em tempo real \\
[Wu et al. 2017] & Sim & CS/GC & OO & Serviços de segurança \\
[Cicirelli et al. 2019] & Sim & GR & OO & Adaptação ao contexto \\
[Kolozali et al. 2018] & Sim & GR & OO & Eventos complexos \\
[Armando et al. 2018] & Sim & GR & UO & Adaptação ao contexto \\
[Tahsien et al. 2020] & Não & GC & UO & Serviços baseado em IA \\
\hline
\end{tabular}

Alguns artigos foram excluídos desta RSL, pois após sua leitura completa foi observada falta de alinhamento com o objeto desta investigação, a saber: [Emmanouilidis et al. 2018], [Singh et al. 2019], apresentam foco em sistemas distribuídos; [Hamrioui et al. 2018], [Gupta et al. 2018], analisam a usabilidade; [Song et al. 2017], [Khamparia et al. 2018], [Wang et al. 2017] restringem sua observação aos aspectos de segurança.

As oportunidades de pesquisa identificadas com a leitura dos artigos e evidenciada 
na tabela 2, quanto aos relacionamentos estabelecidos entre os usuários do ambiente e os objetos, poucos trabalhos enfatizam esta questão. Já nas funcionalidades-chave se percebe pouca atenção à descoberta de serviços e ao gerenciamento de confiança.

Um enfoque arquitetural necessário às soluções SIoT diz respeito a verificação contínua do ambiente, tal aspecto foi bastante observado nos estudos. Para tal, se sugere a extração de dados a partir dos sensores embarcados nos smartphones, bem como a análise das interações realizadas nas redes sociais pelo proprietário desse dispositivo, numa perspectiva de descoberta dos objetos que forneçam os serviços sensíveis ao contexto atual do usuário.

No intuito de aprimoramento dos processos fundamentais das arquiteturas SIoT, os estudos de [Anjomshoa et al. 2017], [Anke 2019], [Cicirelli et al. 2019], [Kolozali et al. 2018] e [Tahsien et al. 2020], integram recursos de Inteligência Artificial (IA) à oferta de serviços oportunos.

Como fechamento desta seção se propõe uma análise baseada nas leituras das produções científicas selecionadas na RSL, onde é possível apontar como principais desafios em funcionalidades-chave para as arquiteturas voltadas a SIoT, os seguintes aspectos: (i) a definição dos círculos sociais das coisas; (ii) o perfil individual do objeto, onde se tem acesso as suas funcionalidades e serviços oferecidos; (iii) o grau de confiança do dispositivo; (iv) a qualificação do serviço prestado; (v) a recomendação gerada pelos outros; (vi) o tempo de resposta oportuno para a maioria destes desafios apresentados.

\section{Considerações Finais}

Os desafios de pesquisa em arquiteturas voltadas à IoT Social, considerando os artigos selecionados nesta revisão sistemática de literatura, sinalizam a importância da composição de serviços em ambientes inteligentes, essa funcionalidade deve estar ajustada às possíveis necessidades dos usuários considerando suas diferentes localizações. Também foram observados esforços de pesquisa em funcionalidades arquiteturais orientadas ao gerenciamento da confiança e dos relacionamentos estabelecidos entre os objetos.

Os obstáculos reportados de forma recorrente nos estudos são intrínsecos dos ambientes computacionais modernos, tais como a elevada mobilidade e a natureza heterogênea dos objetos, os quais estabelecem diferentes graus de relações entre si. Assim, com o intuito de gerar respostas precisas, em menor tempo computacional, muitas propostas sugerem a inserção de elementos de inteligência artificial e processamento mais próximo à borda, estes aspectos são imprescindíveis em futuras soluções arquiteturais da IoT Social.

\section{Referências}

Afzal, B., Umair, M., Shah, G. A., and Ahmed, E. (2019). Enabling iot platforms for social iot applications: vision, feature mapping, and challenges. Future Generation Computer Systems, 92:718-731.

Al-Turjman, F. (2019). 5g-enabled devices and smart-spaces in social-iot: an overview. Future Generation Computer Systems, 92:732-744. 
Anjomshoa, F., Aloqaily, M., Kantarci, B., Erol-Kantarci, M., and Schuckers, S. (2017). Social behaviometrics for personalized devices in the internet of things era. IEEE Access, 5:12199-12213.

Anke, J. (2019). Design-integrated financial assessment of smart services. Electronic Markets, 29(1):19-35.

Armando, N., Rodrigues, A., Pereira, V., Sá Silva, J., and Boavida, F. (2018). An outlook on physical and virtual sensors for a socially interactive internet. Sensors, 18(8):2578.

Bai, L., Yang, D., Wang, X., Tong, L., Zhu, X., Bai, C., and Powell, C. A. (2020). Chinese experts' consensus on the internet of things-aided diagnosis and treatment of coronavirus disease 2019. Clinical eHealth.

Cicirelli, F., Guerrieri, A., Mercuri, A., Spezzano, G., and Vinci, A. (2019). Itema: A methodological approach for cognitive edge computing iot ecosystems. Future Generation Computer Systems, 92:189-197.

Cirani, S., Davoli, L., Ferrari, G., Léone, R., Medagliani, P., Picone, M., and Veltri, L. (2014). A scalable and self-configuring architecture for service discovery in the internet of things. IEEE internet of things journal, 1(5):508-521.

Cumpston, M., Li, T., Page, M. J., Chandler, J., Welch, V. A., Higgins, J. P., and Thomas, J. (2019). Updated guidance for trusted systematic reviews: a new edition of the cochrane handbook for systematic reviews of interventions. Cochrane Database Syst Rev, 10:ED000142.

Dhelim, S., Ning, H., Farha, F., Chen, L., Atzori, L., and Daneshmand, M. (2021). Iot-enabled social relationships meet artificial social intelligence. arXiv preprint arXiv:2103.01776.

Emmanouilidis, C., Bertoncelj, L., Bevilacqua, M., Tedeschi, S., and Ruiz-Carcel, C. (2018). Internet of things-enabled visual analytics for linked maintenance and product lifecycle management. IFAC-PapersOnLine, 51(11):435-440.

Gupta, D., Rodrigues, J. J., Sundaram, S., Khanna, A., Korotaev, V., and de Albuquerque, V. H. C. (2018). Usability feature extraction using modified crow search algorithm: a novel approach. Neural Computing and Applications, pages 1-11.

Gusenbauer, M. (2019). Google scholar to overshadow them all? comparing the sizes of 12 academic search engines and bibliographic databases. Scientometrics, 118(1):177214.

Hamrioui, S., Hamrioui, C. A. M., Lioret, J., and Lorenz, P. (2018). Smart and selforganised routing algorithm for efficient iot communications in smart cities. IET Wireless Sensor Systems, 8(6):305-312.

Iqbal, R., Butt, T. A., Shafique, M. O., Talib, M. W. A., and Umer, T. (2018). Contextaware data-driven intelligent framework for fog infrastructures in internet of vehicles. IEEE Access, 6:58182-58194.

Khamparia, A., Singh, A., Anand, D., Gupta, D., Khanna, A., Kumar, N. A., and Tan, J. (2018). A novel deep learning-based multi-model ensemble method for the prediction of neuromuscular disorders. Neural computing and applications, pages 1-13. 
Kolozali, Ş., Bermudez-Edo, M., FarajiDavar, N., Barnaghi, P., Gao, F., Ali, M. I., Mileo, A., Fischer, M., Iggena, T., Kuemper, D., et al. (2018). Observing the pulse of a city: A smart city framework for real-time discovery, federation, and aggregation of data streams. IEEE Internet of Things Journal, 6(2):2651-2668.

Lippi, M., Mamei, M., Mariani, S., and Zambonelli, F. (2018). An argumentation-based perspective over the social iot. IEEE Internet of Things Journal, 5(4):2537-2547.

Loscri, V., Ruggeri, G., Vegni, A. M., and Cricelli, I. (2018). Social structure analysis in internet of vehicles. In 2018 IEEE International Conference on Sensing, Communication and Networking (SECON Workshops), pages 1-4. IEEE.

Maghawry, N. E. and Ghoniemy, S. (2019). A proposed internet of everything framework for disease prediction. International Journal of Online and Biomedical Engineering (iJOE), 15(04):20-27.

Ning, H., Liu, H., Ma, J., Yang, L. T., and Huang, R. (2016). Cybermatics: Cyberphysical-social-thinking hyperspace based science and technology. Future generation computer systems, 56:504-522.

Petersen, K., Feldt, R., Mujtaba, S., and Mattsson, M. (2008). Systematic mapping studies in software engineering. In Ease, volume 8, pages 68-77.

Rho, S. and Chen, Y. (2019). Social internet of things: Applications, architectures and protocols.

Roopa, M., Pattar, S., Buyya, R., Venugopal, K. R., Iyengar, S., and Patnaik, L. (2019). Social internet of things (siot): Foundations, thrust areas, systematic review and future directions. Computer Communications, 139:32-57.

Saleem, Y., Crespi, N., Rehmani, M. H., Copeland, R., Hussein, D., and Bertin, E. (2016). Exploitation of social iot for recommendation services. In 2016 IEEE 3rd World Forum on Internet of Things (WF-IoT), pages 359-364. IEEE.

Seal, A. and Mukherjee, A. (2018). On the emerging coexistence of edge, fog and cloud computing paradigms in real-time internets-of-everythings which operate in the bigsquared data space. In SoutheastCon 2018, pages 1-9. IEEE.

Singh, S. P., Nayyar, A., Kumar, R., and Sharma, A. (2019). Fog computing: from architecture to edge computing and big data processing. The Journal of Supercomputing, 75(4):2070-2105.

Song, Z., Sun, Y., Yan, H., Wu, D., Niu, P., and Wu, X. (2017). Robustness of smart manufacturing information systems under conditions of resource failure: A complex network perspective. IEEE Access, 6:3731-3738.

Tahsien, S. M., Karimipour, H., and Spachos, P. (2020). Machine learning based solutions for security of internet of things (iot): A survey. Journal of Network and Computer Applications, page 102630.

Wang, K., Du, M., Maharjan, S., and Sun, Y. (2017). Strategic honeypot game model for distributed denial of service attacks in the smart grid. IEEE Transactions on Smart Grid, 8(5):2474-2482.

Wu, J., Su, Z., Wang, S., and Li, J. (2017). Crowd sensing-enabling security service recommendation for social fog computing systems. Sensors, 17(8):1744. 